\title{
Deep Multilingual Correlation for Improved Word Embeddings
}

\author{
Ang Lu ${ }^{1}$, Weiran Wang ${ }^{2}$, Mohit Bansal ${ }^{2}$, Kevin Gimpel ${ }^{2}$, and Karen Livescu ${ }^{2}$ \\ ${ }^{1}$ Department of Automation, Tsinghua University, Beijing, 100084, China \\ lval1@mails.tsinghua.edu.cn \\ ${ }^{2}$ Toyota Technological Institute at Chicago, Chicago, IL, 60637, USA \\ \{weiranwang, mbansal, kgimpel, klivescu\}@ttic.edu
}

\begin{abstract}
Word embeddings have been found useful for many NLP tasks, including part-of-speech tagging, named entity recognition, and parsing. Adding multilingual context when learning embeddings can improve their quality, for example via canonical correlation analysis (CCA) on embeddings from two languages. In this paper, we extend this idea to learn deep non-linear transformations of word embeddings of the two languages, using the recently proposed deep canonical correlation analysis. The resulting embeddings, when evaluated on multiple word and bigram similarity tasks, consistently improve over monolingual embeddings and over embeddings transformed with linear CCA.
\end{abstract}

\section{Introduction}

Learned word representations are widely used in NLP tasks such as tagging, named entity recognition, and parsing (Miller et al., 2004; Koo et al., 2008; Turian et al., 2010; Täckström et al., 2012; Huang et al., 2014; Bansal et al., 2014). The idea in such representations is that words with similar context have similar meaning, and hence should be nearby in a clustering or vector space. Continuous representations are learned with neural language models (Bengio et al., 2003; Mnih and Hinton, 2007; Mikolov et al., 2013) or spectral methods (Deerwester et al., 1990; Dhillon et al., 2011).

The context used to learn these representations is typically the set of nearby words of each word occurrence. Prior work has found that adding translational context results in better representations (Diab and Resnik, 2002; Täckström et al., 2012; Bansal et al., 2012; Zou et al., 2013). Recently, Faruqui and Dyer (2014) applied canonical correlation analysis (CCA) to word embeddings of two languages, and found that the resulting embeddings represent word similarities better than the original monolingual embeddings.

In this paper, we follow the same intuition as Faruqui and Dyer (2014) but rather than learning linear transformations with CCA, we permit the correlated information to lie in nonlinear subspaces of the original embeddings. We use the recently proposed deep canonical correlation analysis (DCCA) technique of Andrew et al. (2013) to learn nonlinear transformations of two languages' embeddings that are highly correlated. We evaluate our DCCA-transformed embeddings on word similarity tasks like WordSim-353 (Finkelstein et al., 2001) and SimLex-999 (Hill et al., 2014), and also on the bigram similarity task of Mitchell and Lapata (2010) (using additive composition), obtaining consistent improvements over the original embeddings and over linear CCA. We also compare tuning criteria and ensemble methods for these architectures.

\section{Method}

We assume that we have initial word embeddings for two languages, denoted by random vectors $\mathbf{x} \in \mathbb{R}^{D_{x}}$ and $\mathbf{y} \in \mathbb{R}^{D_{y}}$, and a set of bilingual word pairs. Our goal is to obtain a representation for each language that incorporates useful information from both $\mathrm{x}$ and y. We consider the two input monolingual word embeddings as different views of the same latent semantic signal. There are multiple ways to incorporate multilingual information into word embeddings. Here we follow Faruqui and Dyer (2014) in taking a CCA-based approach, in which we project the original embeddings onto their maximally correlated subspaces. However, instead of relying on linear correlation, we learn more powerful non-linear transformations of each view via deep networks.

Canonical Correlation Analysis A popular method for multi-view representation learning is canonical correlation analysis (CCA; Hotelling, 1936). Its objective is to find two vectors $\mathbf{u} \in \mathbb{R}^{D_{x}}$ 
and $\mathbf{v} \in \mathbb{R}^{D_{y}}$ such that projections of the two views onto these vectors are maximally (linearly) correlated:

$$
\begin{aligned}
\max _{\mathbf{u} \in \mathbb{R}^{D_{x}}, \mathbf{v} \in \mathbb{R}^{D_{y}}} & \frac{\mathbb{E}\left[\left(\mathbf{u}^{\top} \mathbf{x}\right)\left(\mathbf{v}^{\top} \mathbf{y}\right)\right]}{\sqrt{\mathbb{E}\left[\left(\mathbf{u}^{\top} \mathbf{x}\right)^{2}\right]} \sqrt{\mathbb{E}\left[\left(\mathbf{v}^{\top} \mathbf{y}\right)^{2}\right]}} \\
& =\frac{\mathbf{u}^{\top} \boldsymbol{\Sigma}_{x y} \mathbf{v}}{\sqrt{\mathbf{u}^{\top} \mathbf{\Sigma}_{x x} \mathbf{u}} \sqrt{\mathbf{v}^{\top} \mathbf{\Sigma}_{y y} \mathbf{v}}}
\end{aligned}
$$

where $\boldsymbol{\Sigma}_{x y}$ and $\boldsymbol{\Sigma}_{x x}$ are the cross-view and withinview covariance matrices. (1) is extended to learn multi-dimensional projections by optimizing the sum of correlations in all dimensions, subject to different projected dimensions being uncorrelated. Given sample pairs $\left\{\left(\mathbf{x}_{i}, \mathbf{y}_{i}\right)\right\}_{i=1}^{N}$, the empirical estimates of the covariance matrices are $\hat{\boldsymbol{\Sigma}}_{x x}=$ $\frac{1}{N} \sum_{i=1}^{N} \mathbf{x}_{i} \mathbf{x}_{i}^{\top}+r_{x} \mathbf{I}, \hat{\boldsymbol{\Sigma}}_{y y}=\frac{1}{N} \sum_{i=1}^{N} \mathbf{y}_{i} \mathbf{y}_{i}^{\top}+r_{y} \mathbf{I}$ and $\hat{\boldsymbol{\Sigma}}_{x y}=\frac{1}{N} \sum_{i=1}^{N} \mathbf{x}_{i} \mathbf{y}_{i}^{\top}$ where $\left(r_{x}, r_{y}\right)>0$ are regularization parameters (Hardoon et al., 2004; De Bie and De Moor, 2003). Then the optimal $k$ dimensional projection mappings are given in closed form via the rank- $k$ singular value decomposition $(\mathrm{SVD})$ of the $D_{x} \times D_{y}$ matrix $\hat{\boldsymbol{\Sigma}}_{x x}^{-1 / 2} \hat{\boldsymbol{\Sigma}}_{x y} \hat{\boldsymbol{\Sigma}}_{y y}^{-1 / 2}$.

\subsection{Deep Canonical Correlation Analysis}

A linear feature mapping is often not sufficiently powerful to faithfully capture the hidden, non-linear relationships within the data. Recently, Andrew et al. (2013) proposed a nonlinear extension of CCA using deep neural networks, dubbed deep canonical correlation analysis (DCCA) and illustrated in Figure 1. In this model, two (possibly deep) neural networks $\mathbf{f}$ and $\mathbf{g}$ are used to extract features from each view, and trained to maximize the correlations between outputs in the two views, measured by a linear CCA step with projection mappings $(\mathbf{u}, \mathbf{v})$. The neural network weights and the linear projections are optimized together using the objective

$$
\max _{\mathbf{W}_{\mathbf{f}}, \mathbf{W}_{\mathbf{g}, \mathbf{u}, \mathbf{v}}} \frac{\mathbf{u}^{\top} \boldsymbol{\Sigma}_{f g} \mathbf{v}}{\sqrt{\mathbf{u}^{\top} \boldsymbol{\Sigma}_{f f} \mathbf{u}} \sqrt{\mathbf{v}^{\top} \boldsymbol{\Sigma}_{g g} \mathbf{v}}},
$$

where $\mathbf{W}_{\mathbf{f}}$ and $\mathbf{W}_{\mathbf{g}}$ are the weights of the two networks and $\boldsymbol{\Sigma}_{f g}, \boldsymbol{\Sigma}_{f f}$ and $\boldsymbol{\Sigma}_{g g}$ are covariance matrices computed for $\left\{\mathbf{f}\left(\mathbf{x}_{i}\right), \mathbf{g}\left(\mathbf{y}_{i}\right)\right\}_{i=1}^{N}$ in the same way as CCA. The final transformation is the composition of the neural network and CCA projection, e.g., $\mathbf{u}^{\top} \mathbf{f}(\mathbf{x})$ for the first view. Unlike CCA, DCCA

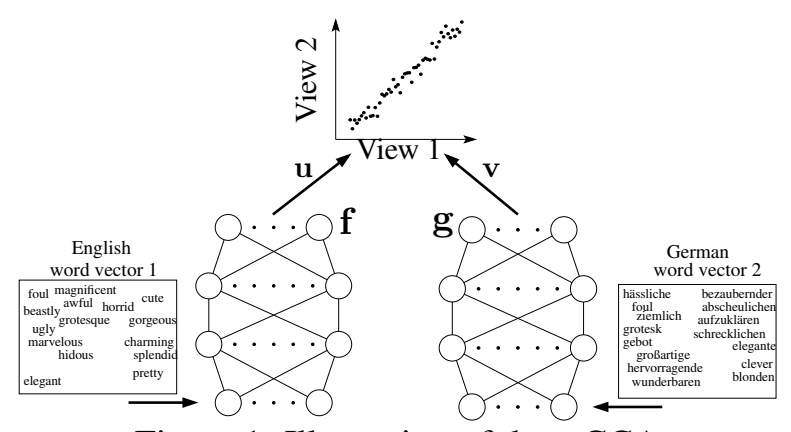

Figure 1: Illustration of deep CCA.

does not have a closed-form solution, but the parameters can be learned via gradient-based optimization, with either batch algorithms like L-BFGS as in (Andrew et al., 2013) or a mini-batch stochastic gradient descent-like approach as in (Wang et al., 2015). We choose the latter in this paper.

An alternative nonlinear extension of CCA is kernel CCA (KCCA) (Lai and Fyfe, 2000; Vinokourov et al., 2003), which introduces nonlinearity through kernels. DCCA scales better with data size, as KCCA involves the SVD of an $N \times N$ matrix. Andrew et al. (2013) showed that DCCA achieves better correlation on held-out data than $\mathrm{CCA} / \mathrm{KCCA}$, and Wang et al. (2015) found that DCCA outperforms CCA and KCCA on a speech recognition task.

\section{Experiments}

We use English and German as our two languages. Our original monolingual word vectors are the same as those used by Faruqui and Dyer (2014). They are 640-dimensional and are estimated via latent semantic analysis on the WMT 2011 monolingual news corpora. ${ }^{1}$ We use German-English translation pairs as the input to CCA and DCCA, using the same set of 36K pairs as used by Faruqui and Dyer. These pairs contain, for each of $36 \mathrm{~K}$ English word types, the single most frequently aligned German word. They were obtained using the word aligner in cdec (Dyer et al., 2010) run on the WMT0610 news commentary corpora and Europarl. After training, we apply the learned CCA/DCCA projection mappings to the original English word embeddings (180K words) and use these transformed embeddings for our evaluation tasks.

\subsection{Evaluation Tasks}

We compare our DCCA-based embeddings to the original word vectors and to CCA-based em-

\footnotetext{
${ }^{1}$ www.statmt.org/wmt11/
} 
beddings on several tasks. We use WordSim353 (Finkelstein et al., 2001), which contains 353 English word pairs with human similarity ratings. It is divided into WS-SIM and WS-REL by Agirre et al. (2009) to measure similarity and relatedness. We also use SimLex-999 (Hill et al., 2014), a new similarity-focused dataset consisting of 666 noun pairs, 222 verb pairs, and 111 adjective pairs. Finally, we use the bigram similarity dataset from Mitchell and Lapata (2010) which has 3 subsets, adjective-noun (AN), noun-noun (NN), and verbobject (VN), and dev and test sets for each. For the bigram task, we simply add the word vectors output by CCA or DCCA to get bigram vectors. ${ }^{2}$

All task datasets contain pairs with human similarity ratings. To evaluate embeddings, we compute cosine similarity between the two vectors in each pair, order the pairs by similarity, and compute Spearman's correlation $(\rho)$ between the model's ranking and human ranking.

\subsection{Training}

We normalize the $36 \mathrm{~K}$ training pair vectors to unit norm (as also done by Faruqui and Dyer). We then remove the per-dimension mean and standard deviation of this set of training pairs, as is typically done in neural network training (LeCun et al., 1998). We do the same to the original 180K English word vectors (normalize to unit norm, remove the mean/standard deviation of the size-36K training set), then apply our CCA/DCCA mappings to these $180 \mathrm{~K}$ vectors. The resulting $180 \mathrm{~K}$ vectors are further normalized to zero mean before cosine similarities between test pairs are computed, as also done by Faruqui and Dyer.

For both CCA and DCCA, we tune the output dimensionality among factors in $\{0.2,0.4,0.6,0.8,1.0\}$ of the original embedding dimension (640), and regularization $\left(r_{x}, r_{y}\right)$ from $\left\{10^{-6}, 10^{-5}, 10^{-4}, 10^{-3}\right\}$, based on the 7 tuning tasks discussed below.

For DCCA, we use standard deep neural networks with rectified linear units and tune the depth ( 1 to 4 hidden layers) and layer widths (in $\{128,256,512,1024,2048,4096\})$ separately for each language. For optimization, we use stochastic

\footnotetext{
${ }^{2}$ We also tried multiplication but it performed worse. In future work, we will directly train on bigram translation pairs.
}

gradient descent (SGD) as described by Wang et al. (2015). We tune SGD hyperparameters on a small grid, choosing a mini-batch size of 3000 , learning rate of 0.0001 , and momentum of 0.99 .

\subsection{Tuning}

Our main results are based on tuning hyperparameters (of CCA/DCCA) on 7 word similarity tasks. ${ }^{3}$ We perform additional experiments in which we tune on the development sets for the bigram tasks. We set aside WS-353, SimLex-999, and the test sets of the bigram tasks as held-out test sets. We consider two tuning criteria:

BestAvg: Choose the hyperparameters with the best average performance across the 7 tuning tasks. This is the only tuning criterion used for CCA.

MostBeat: For DCCA, choose the hyperparameters that beat the best CCA embeddings on a maximum number of the 7 tasks; to break ties here, choose the hyperparameters with the best average performance. The idea is that we want to find a setting that generalizes to many tasks.

We also consider simple ensembles by averaging the cosine similarities from the three best settings under each of these two criteria.

\subsection{Results}

Table 1 shows our main results on the word and bigram similarity tasks. All values are Spearman's correlation $(\rho)$. We show the original word vector results, the best-tuned CCA setting (CCA-1), the ensemble of the top-3 CCA settings (CCA-Ens), and the same for DCCA (with both tuning criteria). The DCCA results show an overall improvement on most tasks over linear CCA (all of the shaded DCCA results are better than all corresponding CCA results).

Each of our tuning criteria for DCCA performs well, and almost always better than CCA. BestAvg is better on some tasks while MostBeat is better on others; we report both here to bring attention to and promote discussion about the effects of tuning methods when learning representations in the absence of supervision or in-domain tuning data.

In Table 2, we report additional bigram similarity results obtained by tuning on the dev sets of the bi-

\footnotetext{
${ }^{3}$ RG-65 (Rubenstein and Goodenough, 1965), MC-30 (Miller and Charles, 1991), MTurk-287 (Radinsky et al., 2011), MTurk-771, MEN (Bruni et al., 2014), Rare Word (Luong et al., 2013), and YP-130 (Yang and Powers, 2006).
} 


\begin{tabular}{|c||ccc||c||cccc||c|}
\hline Embeddings & WS-353 & WS-SIM & WS-REL & SL-999 & AN & NN & VN & Avg & Dim \\
\hline \hline Original & 46.7 & 56.3 & 36.6 & 26.5 & 26.5 & 38.1 & 34.1 & 32.9 & 640 \\
\hline \hline CCA-1 & 67.2 & 73.0 & 63.4 & 40.7 & 42.4 & 48.1 & 37.4 & 42.6 & 384 \\
\hline CCA-Ens & 67.5 & 73.1 & 63.7 & 40.4 & 42.0 & $\mathbf{4 8 . 2}$ & 37.8 & 42.7 & 384 \\
\hline \hline DCCA-1 (BestAvg) & 69.6 & 73.9 & 65.6 & 38.9 & 35.0 & 40.9 & $\mathbf{4 1 . 3}$ & 39.1 & 128 \\
\hline DCCA-Ens (BestAvg) & $\mathbf{7 0 . 8}$ & $\mathbf{7 5 . 2}$ & $\mathbf{6 7 . 3}$ & 41.7 & 42.4 & 45.7 & 40.1 & 42.7 & 128 \\
\hline DCCA-1 (MostBeat) & 68.6 & 73.5 & 65.7 & $\mathbf{4 2 . 3}$ & $\mathbf{4 4 . 4}$ & 44.7 & 36.7 & 41.9 & 384 \\
\hline DCCA-Ens (MostBeat) & 69.9 & 74.4 & 66.7 & $\mathbf{4 2 . 3}$ & 43.7 & 47.4 & 38.8 & $\mathbf{4 3 . 3}$ & 384 \\
\hline
\end{tabular}

Table 1: Main results on word and bigram similarity tasks, tuned on 7 development tasks (see text for details). Shading indicates a result that matches or improves the best linear CCA result; boldface indicates the best result in a given column. See Section 3.4 for discussion on NN results.

\begin{tabular}{|c||cccc|}
\hline Embeddings & AN & NN & VN & Avg \\
\hline CCA & 42.4 & $\mathbf{4 8 . 1}$ & 37.4 & 42.6 \\
Deep CCA & $\mathbf{4 5 . 5}$ & 47.1 & $\mathbf{4 5 . 1}$ & $\mathbf{4 5 . 9}$ \\
\hline
\end{tabular}

Table 2: Bigram results, tuned on bigram dev sets.

gram tasks themselves (as provided by Mitchell and Lapata), since the 7 tuning tasks are not particularly related to the bigram test sets. We see that DCCA can achieve even stronger improvements over CCA and overall using these related dev sets.

We note that the performance on the $\mathrm{NN}$ task does not improve. The typical variance of annotator scores for each bigram pair was larger for the $\mathrm{NN}$ dataset than for the other bigram datasets, suggesting noisier annotations. Also, we found that the $\mathrm{NN}$ annotations often reflected topical relatedness rather than functional similarity, e.g., television set and television programme are among the most similar noun-noun bigrams. We expect that multilingual information would help embeddings to more closely reflect functional similarity.

For DCCA, we found that the best-performing networks were typically asymmetric, with 1 to 2 layers on the English side and 2 to 4 on the German side. The best network structure on the bigram VN development set is 640-128-128 for the English view and 640-128-512-128 for the German view, with a final CCA projection layer with dimensionality 128 for each language.

\section{Discussion}

Normalization and Evaluation We note that the cosine similarity (and thus Spearman's $\rho$ ) between a pair of words is not invariant to the series of simple (affine) transformations done by the normalizations in our procedure. For their baseline, Faruqui and Dyer (2014) did not remove the standard deviation

\begin{tabular}{|rl|rl|}
\hline \multicolumn{2}{|c|}{ better with DCCA } & worse with DCCA \\
\hline arrive & come & author & creator \\
locate & find & leader & manager \\
way & manner & buddy & companion \\
recent & new & crowd & bunch \\
take & obtain & achieve & succeed \\
boundary & border & attention & interest \\
win & accomplish & join & add \\
contemplate & think & mood & emotion \\
\hline
\end{tabular}

Table 3: Highly-similar pairs in SimLex-999 that improved/degraded the most under DCCA. Pairs are sorted in decreasing order according to the amount of improvement/degradation.

of the $36 \mathrm{~K}$ training set for the $180 \mathrm{~K}$ English vocabulary during testing. We have accidentally found that this normalization step alone greatly improves the performance of the original vectors.

For example, the WS-353 correlation improves from 46.7 to 67.1 , essentially matching the linear CCA correlations, though DCCA still outperforms them both. This indicates that the cosine similarity is not stable, and it is likely better to learn a distance/similarity function (using labeled tuning data) atop the learned features such that similarities between selected pairs will match the human similarities, or such that the rankings will match.

Error Analysis We analyze high-similarity word pairs that change the most with DCCA, as compared to both linear CCA and the original vectors.

For a word pair $w$, we use $r(w)$ to refer to its similarity rank, subscripting it whether it is computed according to human ratings $\left(r_{h}\right)$ or if based on cosine similarity via the original vectors $\left(r_{o}\right)$, CCA-1 $\left(r_{c}\right)$, or DCCA-1 MostBeat $\left(r_{d}\right)$. We define $\delta_{a}(w)=\left|r_{a}(w)-r_{h}(w)\right|$ and compute $\Delta(w)=$ 


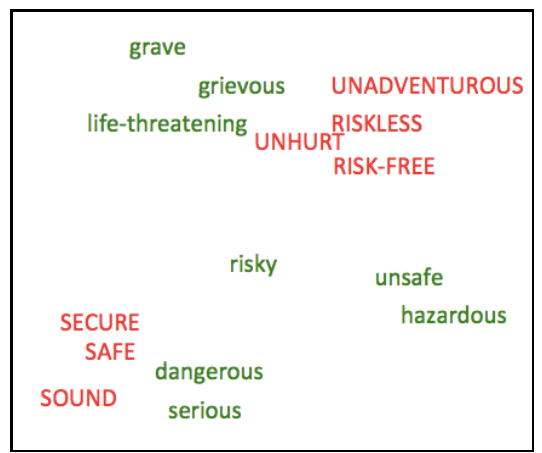

Original

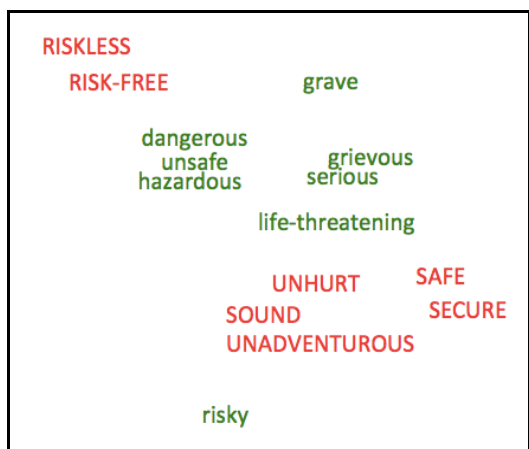

CCA-1

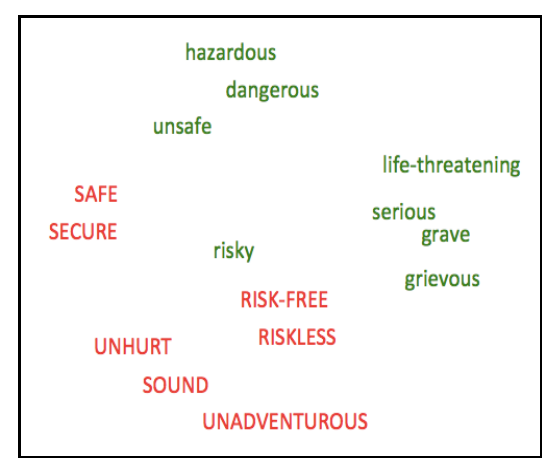

DCCA-1 (MostBeat)

Figure 2: t-SNE visualization of synonyms (green) and antonyms (red, capitalized) of dangerous.

$\delta_{d}(w)-\left(\delta_{c}(w)+\delta_{o}(w)\right)$. If $\Delta(w)<0$, then word pair $w$ was closer to the human ranking using DCCA. Table 3 shows word pairs from SimLex-999 with high human similarity ratings ( $\geq 7$ out of 10 ); column 1 shows pairs with smallest $\Delta$ values, and column 2 shows pairs with largest $\Delta$ values.

Among pairs in column 1, many contain words with several senses. Using bilingual information is likely to focus on the most frequent sense in the bitext, due to our use of the most frequently-aligned German word in each training pair. By contrast, using only monolingual context is expected to find an embedding that blends the contextual information across all word senses.

Several pairs from column 2 show hypernym rather than paraphrase relationships, e.g., authorcreator and leader-manager. Though these pairs are rated as highly similar by annotators, linear CCA made them less similar than the original vectors, and DCCA made them less similar still. This matches our intuition that bilingual information should encourage paraphrase-like similarity and thereby discourage the similarity of hypernym-hyponym pairs.

Visualizations We visualized several synonymantonym word lists and often found that DCCA more cleanly separated synonyms from antonyms than CCA or the original vectors. An example of the clearest improvement is shown in Fig. 2.

\section{Related work}

Previous work has successfully used translational context for word representations (Diab and Resnik, 2002; Zhao et al., 2005; Täckström et al., 2012; Bansal et al., 2012; Faruqui and Dyer, 2014), including via hand-designed vector space models (Peirsman and Padó, 2010; Sumita, 2000) or via unsuper- vised LDA and LSA (Boyd-Graber and Blei, 2009; Zhao and Xing, 2006).

There have been other recent deep learning approaches to bilingual representations, e.g., based on a joint monolingual and bilingual objective (Zou et al., 2013). There has also been recent interest in learning bilingual representations without using word alignments (Chandar et al., 2014; Gouws et al., 2014; Kočiskỳ et al., 2014; Vulic and Moens, 2013).

This research is also related to early examples of learning bilingual lexicons using monolingual corpora (Koehn and Knight, 2002; Haghighi et al., 2008); the latter used CCA to find matched word pairs. Irvine and Callison-Burch (2013) used a supervised learning method with multiple monolingual signals. Finally, other work on CCA and spectral methods has been used in the context of other types of views (Collobert and Weston, 2008; Dhillon et al., 2011; Klementiev et al., 2012; Chang et al., 2013).

\section{Conclusion}

We have demonstrated how bilingual information can be incorporated into word embeddings via deep canonical correlation analysis (DCCA). The DCCA embeddings consistently outperform linear CCA embeddings on word and bigram similarity tasks. Future work could compare DCCA to other nonlinear approaches discussed in $\S 5$, compare different languages as multiview context, and extend to aligned phrase pairs, and to unaligned data.

\section{Acknowledgments}

We are grateful to Manaal Faruqui for sharing resources, and to Chris Dyer, David Sontag, Lyle Ungar, and anonymous reviewers for helpful input. 


\section{References}

Eneko Agirre, Enrique Alfonseca, Keith Hall, Jana Kravalova, Marius Pacsca, and Aitor Soroa. 2009. A study on similarity and relatedness using distributional and wordnet-based approaches. In Proceedings of HLT-NAACL.

Galen Andrew, Raman Arora, Jeff Bilmes, and Karen Livescu. 2013. Deep canonical correlation analysis. In Proceedings of ICML.

Mohit Bansal, John DeNero, and Dekang Lin. 2012. Unsupervised translation sense clustering. In Proceedings of NAACL-HLT.

M. Bansal, K. Gimpel, and K. Livescu. 2014. Tailoring continuous word representations for dependency parsing. In Proceedings of ACL.

Yoshua Bengio, Réjean Ducharme, Pascal Vincent, and Christian Janvin. 2003. A neural probabilistic language model. JMLR, 3:1137-1155, March.

Jordan Boyd-Graber and David M Blei. 2009. Multilingual topic models for unaligned text. In Proceedings of UAI.

Elia Bruni, Nam-Khanh Tran, and Marco Baroni. 2014. Multimodal distributional semantics. J. Artif. Intell. Res.(JAIR), 49:1-47.

Sarath Chandar, Stanislas Lauly, Hugo Larochelle, Mitesh Khapra, Balaraman Ravindran, Vikas Raykar, and Amrita Saha. 2014. An autoencoder approach to learning bilingual word representations. In Proceeddings of NIPS.

Kai-Wei Chang, Wen-tau Yih, and Christopher Meek. 2013. Multi-relational latent semantic analysis. In Proceedings of EMNLP.

Ronan Collobert and Jason Weston. 2008. A unified architecture for natural language processing: Deep neural networks with multitask learning. In Proceedings of ICML.

Tijl De Bie and Bart De Moor. 2003. On the regularization of canonical correlation analysis. Int. Sympos. ICA and BSS, pages 785-790.

Scott C. Deerwester, Susan T. Dumais, Thomas K. Landauer, George W. Furnas, and Richard A. Harshman. 1990. Indexing by latent semantic analysis. JASIS, 41(6):391-407.

Paramveer Dhillon, Dean P. Foster, and Lyle H. Ungar. 2011. Multi-view learning of word embeddings via CCA. In Proceedings of NIPS.

Mona Diab and Philip Resnik. 2002. An unsupervised method for word sense tagging using parallel corpora. In Proceedings of ACL.

C. Dyer, A. Lopez, J. Ganitkevitch, J. Weese, F. Ture, P. Blunsom, H. Setiawan, V. Eidelman, and P. Resnik. 2010. cdec: A decoder, alignment, and learning framework for finite-state and context-free translation models. In Proceedings of ACL.

Manaal Faruqui and Chris Dyer. 2014. Improving vector space word representations using multilingual correlation. In Proceedings of EACL.

Lev Finkelstein, Evgeniy Gabrilovich, Yossi Matias, Ehud Rivlin, Zach Solan, Gadi Wolfman, and Eytan Ruppin. 2001. Placing search in context: The concept revisited. In Proceedings of $W W W$.

Stephan Gouws, Yoshua Bengio, and Greg Corrado. 2014. Bilbowa: Fast bilingual distributed representations without word alignments. arXiv preprint arXiv:1410.2455.

Aria Haghighi, Percy Liang, Taylor Berg-Kirkpatrick, and Dan Klein. 2008. Learning bilingual lexicons from monolingual corpora. In Proceedings of ACL.

David R. Hardoon, Sandor Szedmak, and John ShaweTaylor. 2004. Canonical correlation analysis: An overview with application to learning methods. Neural Computation, 16(12):2639-2664, December.

Felix Hill, Roi Reichart, and Anna Korhonen. 2014. Simlex-999: Evaluating semantic models with (genuine) similarity estimation. arXiv preprint arXiv:1408.3456.

Harold Hotelling. 1936. Relations between two sets of variates. Biometrika, 28(3/4):321-377, December.

Fei Huang, Arun Ahuja, Doug Downey, Yi Yang, Yuhong Guo, and Alexander Yates. 2014. Learning representations for weakly supervised natural language processing tasks. Computational Linguistics, 40(1).

Ann Irvine and Chris Callison-Burch. 2013. Supervised bilingual lexicon induction with multiple monolingual signals. In Proceedings of HLT-NAACL, pages 518523.

Alexandre Klementiev, Ivan Titov, and Binod Bhattarai. 2012. Inducing crosslingual distributed representations of words.

Tomáš Kočiskỳ, Karl Moritz Hermann, and Phil Blunsom. 2014. Learning bilingual word representations by marginalizing alignments. arXiv preprint arXiv:1405.0947.

Philipp Koehn and Kevin Knight. 2002. Learning a translation lexicon from monolingual corpora. In Proceedings of the ACL Workshop on Unsupervised Lexical Acquisition.

Terry Koo, Xavier Carreras, and Michael Collins. 2008. Simple semi-supervised dependency parsing. In Proceedings of ACL.

P. L. Lai and C. Fyfe. 2000. Kernel and nonlinear canonical correlation analysis. Int. J. Neural Syst., 10(5):365-377, October.

Yann LeCun, Leon Bottou, Genevieve B. Orr, and KlausRobert Müller. 1998. Efficient backprop. volume 
1524 of Lecture Notes in Computer Science, pages 950, Berlin. Springer-Verlag.

Minh-Thang Luong, Richard Socher, and Christopher D. Manning. 2013. Better word representations with recursive neural networks for morphology. In Proceedings of CoNLL.

Tomas Mikolov, Ilya Sutskever, Kai Chen, Greg Corrado, and Jeffrey Dean. 2013. Distributed representations of words and phrases and their compositionality. In Proceedings of NIPS.

George A Miller and Walter G Charles. 1991. Contextual correlates of semantic similarity. Language and cognitive processes, 6(1):1-28.

Scott Miller, Jethran Guinness, and Alex Zamanian. 2004. Name tagging with word clusters and discriminative training. In Proceedings of HLT-NAACL.

Jeff Mitchell and Mirella Lapata. 2010. Composition in distributional models of semantics. Cognitive Science, 34(8):1388-1439.

Andriy Mnih and Geoffrey Hinton. 2007. Three new graphical models for statistical language modelling. In Proceedings of ICML.

Yves Peirsman and Sebastian Padó. 2010. Crosslingual induction of selectional preferences with bilingual vector spaces. In Proceedings of HLT-NAACL.

Kira Radinsky, Eugene Agichtein, Evgeniy Gabrilovich, and Shaul Markovitch. 2011. A word at a time: computing word relatedness using temporal semantic analysis. In Proceedings of $W W W$.

Herbert Rubenstein and John B Goodenough. 1965. Contextual correlates of synonymy. Communications of the ACM, 8(10):627-633.

Eiichiro Sumita. 2000. Lexical transfer using a vectorspace model. In Proceedings of ACL.

Oscar Täckström, Ryan McDonald, and Jakob Uszkoreit. 2012. Cross-lingual word clusters for direct transfer of linguistic structure. In Proceedings of HLT-NAACL.

Joseph Turian, Lev-Arie Ratinov, and Yoshua Bengio. 2010. Word representations: A simple and general method for semi-supervised learning. In Proceedings of $A C L$.

Alexei Vinokourov, Nello Cristianini, and John ShaweTaylor. 2003. Inferring a semantic representation of text via cross-language correlation analysis. In Proceedings of NIPS.

Ivan Vulic and Marie-Francine Moens. 2013. A study on bootstrapping bilingual vector spaces from nonparallel data (and nothing else). In Proceedings of EMNLP.

Weiran Wang, Raman Arora, Karen Livescu, and Jeff Bilmes. 2015. Unsupervised learning of acoustic features via deep canonical correlation analysis. In Proceedings of ICASSP.
Dongqiang Yang and David MW Powers. 2006. Verb similarity on the taxonomy of wordnet. Proceedings of GWC-06, pages 121-128.

Bing Zhao and Eric P Xing. 2006. Bitam: Bilingual topic admixture models for word alignment. In Proceedings of the COLING/ACL on Main conference poster sessions, pages 969-976. ACL.

Bing Zhao, Eric P Xing, and Alex Waibel. 2005. Bilingual word spectral clustering for statistical machine translation. In Proceedings of the ACL Workshop on Building and Using Parallel Texts.

Will Y Zou, Richard Socher, Daniel M Cer, and Christopher D Manning. 2013. Bilingual word embeddings for phrase-based machine translation. In Proceedings of EMNLP. 